\title{
Engineering Chemistry of Electrospun Nanofibers and Interfaces in Nanocomposites for Superior Mechanical Properties
}

\author{
Elif Özden, Yusuf Z. Menceloğlu, and Melih Papila*
}

Sabanc1 University, Materials Science and Engineering, Advanced Composites and Polymer Processing Laboratory, 34956 Tuzla, Istanbul, Turkey

\begin{abstract}
The novelty of this work is based on designing the chemistry of the electrospun nanofibers, so that the resultant composites substantially benefit from cross-linking between the nanofibers and the polymer matrix. Specifically, the solution of in-house synthesized copolymers polystyrene-co-glycidyl methacrylate P(St-co-GMA) is electrospun to produce mats of surface reactive nanoto-submicron scale fibers that are accompanied later by spraying over the ethylenediamine (EDA) as a supplementary cross-linking agent for epoxy. The P(St-co-GMA)/EDA fiber mats are then embedded into an epoxy resin. Analysis of the three-point-bending mode of the composites reveals that the storage modulus of $\mathrm{P}(\mathrm{St}-\mathrm{CO}-\mathrm{GMA}) / \mathrm{EDA}$ nanofiber-reinforced epoxy are about 10 and 2.5 times higher than that of neat and P(St-co-GMA) nanofiber-reinforced epoxy, respectively, even though the weight fraction of the nanofibers was as low as $2 \mathrm{wt} \%$. The significant increase in the mechanical response is attributed to the inherently cross-linked fiber structure and the surface modification/chemistry of the electrospun fibers, that results in cross-linked polymer matrix-nanofiber interfacial bonding.
\end{abstract}

KEYWORDS: electrospinning $\bullet$ nanofiber $\bullet$ composite $\bullet$ surface modification $\bullet$ cross-linked interface

\section{INTRODUCTION}

N anoscaled constituents in composites are of interest because of their potential for significantly improving the composite material properties $(1-7)$. Nano- to submicrometer-scale polymeric fibers formed by electrospinning, for instance, have recently been explored for their reinforcing ability in composites (8-19). By forming a network of the fibers, electrospinning secures the uniform planar dispersion of the fibers that can be preserved when used in polymeric matrix composite materials (20). The process also results in a large draw ratio, causing extended chain conformations and highly crystalline regions of polymer structure in favor of fiber mechanical properties (21). The electrospun polymeric fibers were utilized as reinforcement to enhance particularly the matrix-dominated flexural properties of cross-linked polymer matrix composites (18-22). Recently, cellulose, nylon 4,6, carbon nanofiber, polyvinyl alcohol (PVOH), poly(l-lactide) (PLLA), polyacrylonitrile (PAN), polymethyl-methacrylate (PMMA) polymeric, and nanoscaled glass electrospun fibers were successfully employed to reinforce a polymer matrix (8-18). It was demonstrated (23) that strong interfacial bonding has been crucial to benefit from the unique properties of nanofibers for composite reinforcement. Since nanoscaled materials have enormous surface area, interfacial sliding of the nanoscale fillers in the polymeric matrices may result in an extremely efficient mechanism for damping enhancement (23). Additionally, strong surface interactions enable good mechan-

* Corresponding author.

Received for review March 31, 2010 and accepted June 03, 2010

DOI: $10.1021 / \mathrm{am} 100288 \mathrm{r}$

2010 American Chemical Society ical interlocking with surrounding polymer chains (24), thereby strengthening the nanocomposites. Hence, several researchers $(8,9,13,25)$ have studied the importance of interfacial bonding to obtain better mechanical performance, due to nanostructures as composite reinforcements. However, to the best of author's knowledge, investigations specific to the cross-linked nanofiller-matrix interface for better interfacial bonding are still needed.

In this study, the hypothesis is that incorporation of electrospun surface modified/reactive nanofibers with epoxide functional groups into the epoxy resin results in significant improvements in the mechanical properties. To investigate this hypothesis, along with polystyrene (PSt) nanofibers, we also produced surface-activation capable polystyrene-co-glycidyl methacrylate P(St-co-GMA) nanofibers by electrospinning. The surface chemistry of these fibers is expected to improve interfacial bonding with the epoxy based polymer matrix, as the glycidylmethacrylate (GMA) structure contains epoxide ring-promoting crosslinking across the interface. The experimental procedure was designed to explore the effects of the presence of nanofibrous layers, the GMA composition in the fiber chemical structure and supplement by a cross-linking agent (ethylenediamine, EDA) that was applied onto the fibers by spraying, prior to embedding the fibrous mats into epoxy matrix. These factors, the chemistry or the functional groups of the nanofibers and cross-linking agent, were investigated primarily for the mechanical response and thermal stability of the polymer nanofiber-reinforced epoxy matrix composites.

\section{EXPERIMENTAL SECTION}

Copolymer Synthesis. The monomers styrene (purified) and glycidylmethacrylate (GMA) were supplied by 


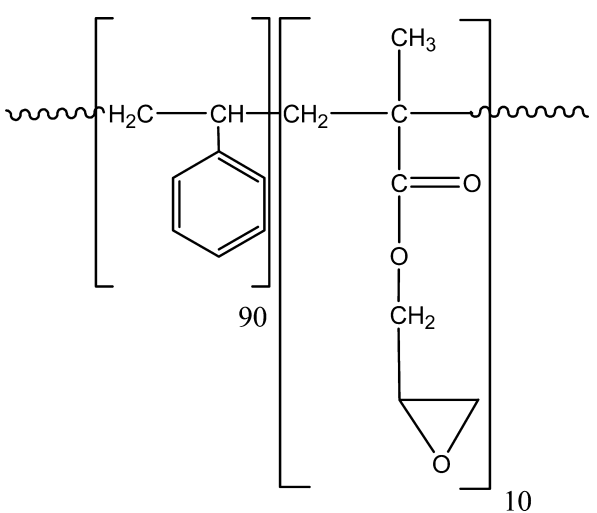

FIGURE 1. Chemical Structure of P(St-co-GMA).

Aldrich Chemical Co, whereas the solvents, $N, N$ dimethylformamide and methanol, were purchased from Merck Chemicals Co. Copolymer PSt-co-GMA were synthesized by solution polymerization technique as well as polystyrene. Purified styrene and GMA (by weight fractions: $90 \%$ St and $10 \%$ GMA) were put into a test tube in an ice bath. Dimethylformamide (DMF) was then added into St-GMA monomer mix such that volume proportion is $3: 2$, respectively. The initiator azobisisobutyronitrile (AIBN) was added into the test tube flushed with nitrogen. The tube containing the dissolved monomers was then kept $24 \mathrm{~h}$ in the constant temperature bath at $65^{\circ} \mathrm{C}$ for the polymerization reaction. Finally, the polymer solution was poured out into a beaker containing methanol and the methanol/polymer mixture was filtered and dried in an oven at $60{ }^{\circ} \mathrm{C}$ for $2 \mathrm{~h}$. The synthesized P(Stco-GMA) copolymer structure (see Figure 1) was determined by $\mathrm{H}$ NMR. Molecular weights and polydispersities were measured by a gel permeation chromatography (GPC) system and the range was recorded as 110000 and 160000 $\mathrm{g} / \mathrm{mol}$. (1.35-1.45 PDI).

Electrospinning of PSt and P(St-co-GMA) Nanofibers. Polymer solutions PSt/DMF and (P(St-Co-GMA))/DMF, at $30 \mathrm{wt} \%$ polymer concentration, were prepared at room temperature. The solutions were stirred magnetically for $24 \mathrm{~h}$ to obtain homogeneity and then electrospun to produce the nonwoven fiber mats. Schematic instrumentation of the electrospinning setup is shown in Figure 2. An electrical bias potential (via Gamma High Voltage ES 30P-20W) was applied to the polymer solutions contained in $2 \mathrm{~mL}$ syringe, which has an alligator clip attached to the syringe needle (diameter $300 \mu \mathrm{m})$. The applied voltage was adjusted to $15 \mathrm{kV}$, while the grounded collector covered with aluminum foil was placed $10 \mathrm{~cm}$ away from the syringe needle. A syringe pump (NewEra NE-1000 Syringe Pump) was used to maintain a solution flow rate of $30 \mu \mathrm{L} / \mathrm{h}$ during electrospinning.

Cross-Linking of P(St-co-GMA) Nanofibers. An extra set of P(St-Co-GMA) fiber mats was treated by spraying ethylenediamine (EDA) (nominal mass fraction of nanofiber: EDA is up to 1:4) to facilitate self-cross-linking of nanofibers and chemical interaction of epoxide "resin" with the polyamine "hardener" (see Figure 3). Inherent cross-linking in these fibers of tuned chemistry, is called hereafter as P(St-co-GMA)/ EDA fibers. Sol-gel analysis was performed to determine the degree of cross-linking in the P(St-co-GMA)/EDA fibers

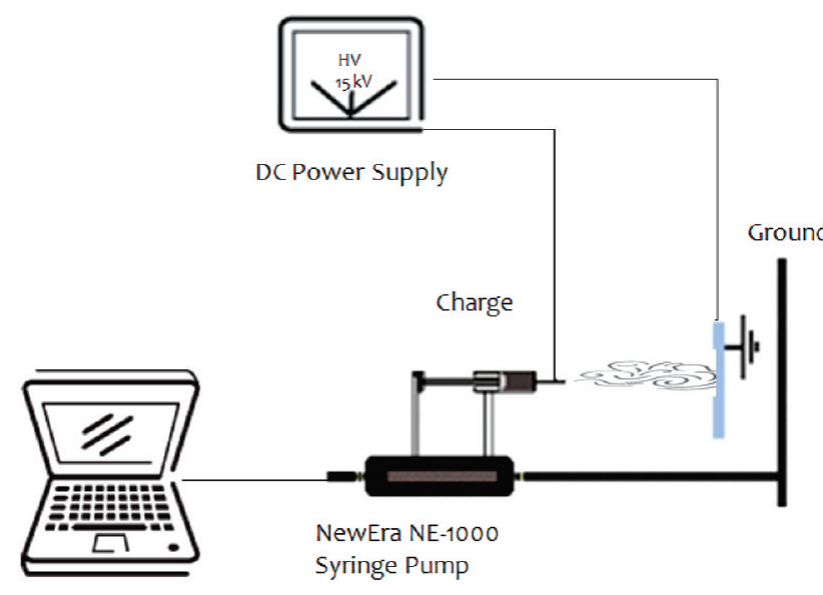

New Era Pump Term

FIGURE 2. Illustration of electrospinning setup.

using two different solvents, DMF and acetone. The crosslinked fibers were put in the solvent and kept soaked for 3 days at room temperature. The swollen fibers were then dried. Gel fraction as a measure of the cross-linking was calculated as follows

$$
\begin{aligned}
& \% \text { sol fraction }=\left[\left(\left(m_{\mathrm{i}}-m_{\mathrm{f}}\right) / m_{\mathrm{i}}\right) 100\right] \\
& \% \text { gel fraction }=100-\% \text { sol fraction }
\end{aligned}
$$

where $m_{\mathrm{f}}$ is the dry mass of the extracted sample and $m_{\mathrm{i}}$ is the initial mass of the sample (26). The analyses resulted in a range of $68 \%-71 \%$ gel fraction of cross-linked fibers, whereas PSt-co-GMA fibers were completely soluble in DMF before the EDA spraying. It should also be noted that addition of cross-linking agent directly to the polymer solution prior to electrospinning was also done. However, immediate changes in the solution characteristics due to triggered cross-linking prevented the production of fibers of the desired characteristics.

Fabrication of Nanofiber Reinforced Composites for DMA Testing. Sets of cross-linked P(St-co-GMA)/ EDA fibers, along with PSt and P(St-co-GMA) as received fibers, were first cut into $12 \mathrm{~mm} \times 50 \mathrm{~mm}$ pieces. The thickness of the electrospun fiber mat layer is approximately $25 \mu \mathrm{m}$. Next, the fiber mats were embedded into epoxy resin (Hunstman Adv. Mat. Co. Araldite LY 564 and XB 3403) layer by layer, using a Teflon mold custom-designed for the net-shape of DMA specimen. The epoxy matrix composites reinforced by 10 layers of the fiber webs (corresponding approximately $2 \%$ fiber weight fraction) were cured at $50^{\circ}$ for $15 \mathrm{~h}$ and DMA specimens of size $2 \mathrm{~mm} \times 12 \mathrm{~mm} \times 50$ $\mathrm{mm}$ were obtained. Note that the fiber weight fraction of $2 \%$ here is a representative amount for the proof of cross-linking fiber-matrix interface concept, but the fiber content is an important factor to look into in future studies.

Characterization of the Electrospun Fibers and Composites. Glass transition temperatures $\left(T_{\mathrm{g}}\right)$ of the nanofibers and nanofiber-reinforced composites were de- 
A

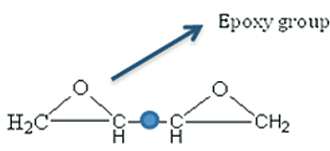

Protopolymer

B

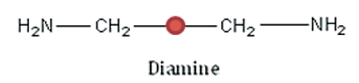

C

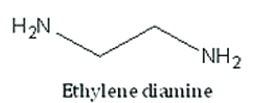

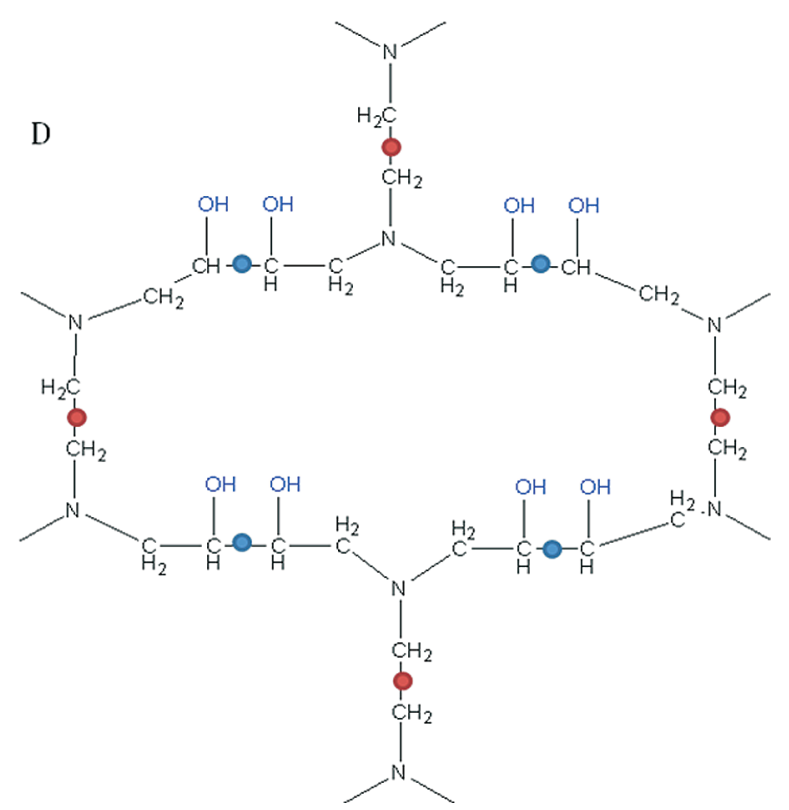

FIGURE 3. Chemical structure of (A) epoxy resin, (B) hardener, (C) cross-linking agent ethylene diamine, and (D) cross-linked network of epoxy.

termined by using a differential scanning analyzer (Netzsch DSC 204) and dynamic mechanical thermal analyzer (Netzsch DMA 242), respectively. Morphologies of PSt, P(St-co-GMA) and P(St-co-GMA)/EDA fibrous webs and fracture surfaces of the neat epoxy and nanofiber-reinforced composites were evaluated by scanning electron microscopy containing field emission gun (SEM LEO 1530VP) using secondary electron detector at $2 \mathrm{kV}$. Both the electrospun mats and nanofiberreinforced composites were carbon coated for better electrical conduction. The DMA tests of the neat epoxy and nanofiber-reinforced composites were performed in three point-bending mode at a frequency of $1 \mathrm{~Hz}$ over a temperature range of $20-90{ }^{\circ} \mathrm{C}$. Testing limits on amplitude, maximum dynamic force, and static constant force were set as $30 \mu \mathrm{m}, 3 \mathrm{~N}$, and $0.01 \mathrm{~N}$, respectively. Ten samples were tested for each of the three fiber types. Finally, a universal testing machine (UTM, ZWICK Proline Z100) was used to determine flexural strength and flexural modulus at room temperature using the ASTM D790 standard.

\section{RESULTS AND DISCUSSION}

It is vital to confirm that electrospinning of the polymer solutions resulted in fibrous formation, based on the selected processing parameters. The morphologies of PSt and P(Stco-GMA) electrospun fibrous mats are shown in the SEM images in Figure 4A and B. The images demonstrate that bead-free fiber formation was achieved, and the diameter of PSt and P(St-co-GMA) fibers is in the range of $200 \mathrm{~nm}$ to $1 \mu \mathrm{m}$. The variance in the fiber diameter is rather high and calls for a systematic study aiming for optimal process conditions of the minimal diameter and variance. A designof-experiments-based study similar to our earlier work (27) is also underway.

The SEM micrograph of $\mathrm{P}(\mathrm{St}-\mathrm{co}-\mathrm{GMA}) / \mathrm{EDA}$ ribbonlike fibers (Figure 4C) demonstrates that cross-linking was induced by the spraying of EDA on P(St-co-GMA) fibers. The cross-linked fiber diameter was in the range of $400 \mathrm{~nm}$ to 2 $\mu \mathrm{m}$ because of swelling caused by ethylenediamine. These changes on the morphology and solubility tests suggest that a high degree of cross-linking occurred, around $70 \%$.

As primary objective in this work is to enhance the interface performance by designing or engineering the surface chemistry of electrospun fibers, it is essential to assess the interface-related properties. The damping ratio or loss tangent curves by DMA, can be considered as one of the metrics for improved interfacial bonding. The damping ratio $(\tan \delta)$ reflects the ability of the material to dissipate energy and in the case of composite or multiphase materials interaction between the inner phases and interfaces can dominate the energy dissipation $(24,28-31)$. The energy loss at the interface depends on the product of applied internal forces and the slipping displacement (32). Considering the inversely proportional influence of the interfacial strength on the slipping displacement, surface-modified nanofiber-reinforced composites with enhanced interfacial bonding is anticipated to result in smaller slipping displacement. Thus, the energy dissipation is reduced and damping ratio is decreased. The reduction is evident by $\tan \delta$ comparison among the electrospun fiber-reinforced composites in Figure 5. The damping ratio of PSt nanofiber-reinforced composites, for instance, was higher than that of $\mathrm{P}(\mathrm{St}-\mathrm{CO}-$ GMA) nanofiber-reinforced composites for which the fibermatrix interface is improved and stronger. The curve associated with the P(St-Co-GMA)/EDA nanofibers is the broadest with the lowest amplitude, an indication of the improved compatibility or interface with the polymer matrix (8). On the other hand, the three types of embedded fiber mats here resulted in substantial increase in stiffness at a cost of damping ratio compared to the neat epoxy (Figures 5 and 6). The reinforcing and stiffening effect due to superior adhesion and load transfer between nanofibers and epoxy 


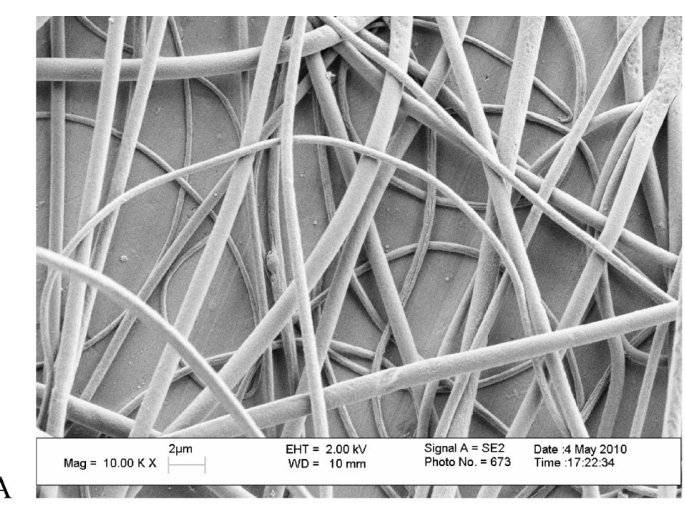

A
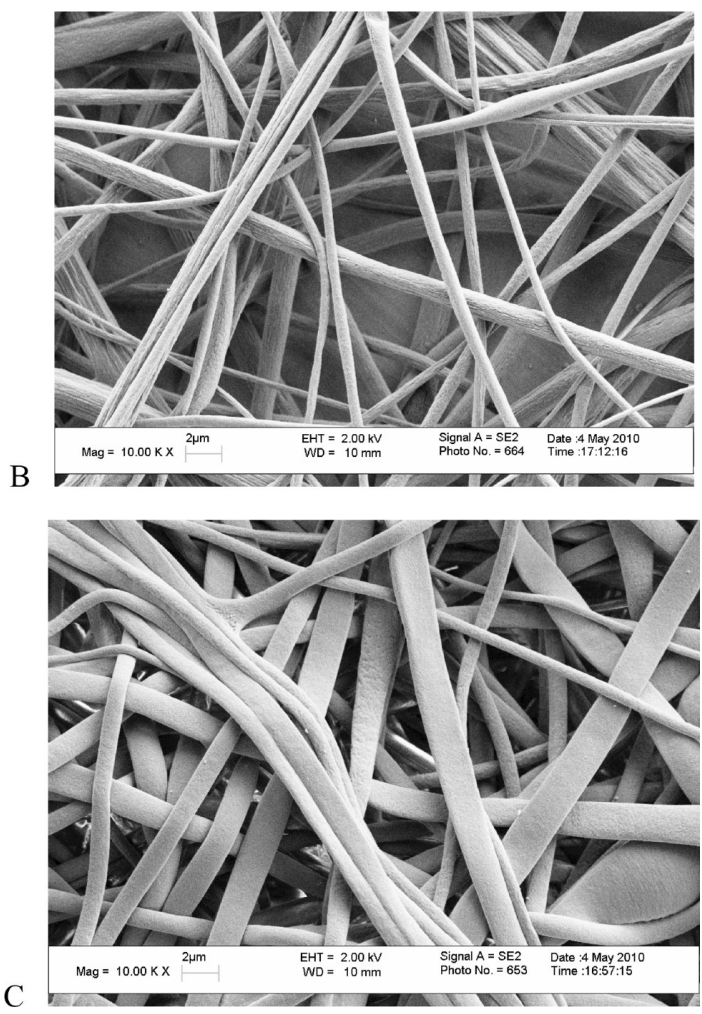

FIGURE 4. SEM micrographs of fibers within the fiber diameter range (A) PSt nanofibers in $300 \mathrm{~nm}$ to $1 \mu \mathrm{m}, T_{\mathrm{g}} 105^{\circ} \mathrm{C}$; (B) P(St-co-GMA) nanofibers in $200 \mathrm{~nm}$ to $1 \mu \mathrm{m}, T_{\mathrm{g}} 96^{\circ} \mathrm{C}$; and (C) P(St-co-GMA)/EDA nanofibers in $400 \mathrm{~nm}$ to $2 \mu \mathrm{m}, T_{\mathrm{g}} 121{ }^{\circ} \mathrm{C}$,

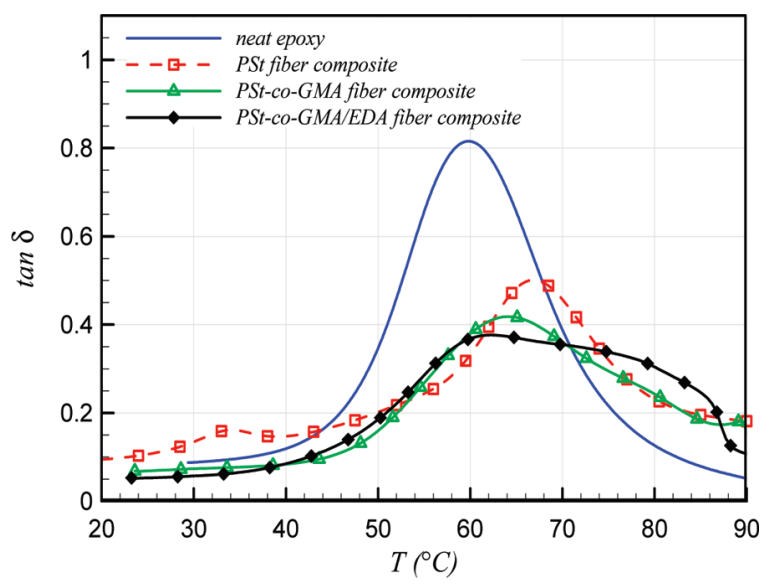

FIGURE 5. Damping ratio, $\tan \delta$, vs temperature of reinforced and unreinforced epoxy specimens.

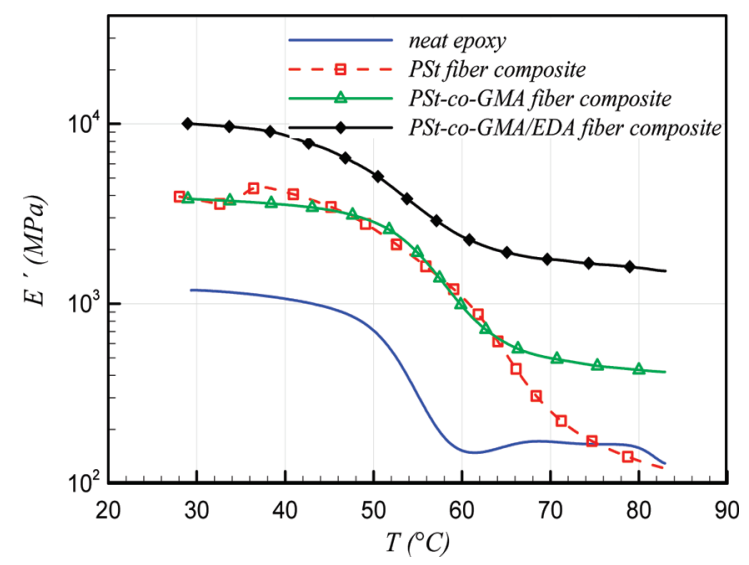

FIGURE 6. Storage modulus vs temperature, reinforcement with P(Stco-GMA) with/without amine-sprayed nanofiber, and PSt nanofiberreinforced composites compared to neat epoxy.

matrix appear to override the potential damping ratio enhancement due to interfacial interactions in nanocomposites when compared to the neat polymer matrix. The reinforcement-damping trade-off reported here is also consistent with the literature, such as the results presented by Suhr et al. (24), for instance, on silica particle-reinforced/ stiffened nanocomposites.

Table 1 summarizes the glass-transition temperature $T_{\mathrm{g}}$, and loss tangent, $\tan \delta$ determined by DMA ( $T_{g}$ is considered herein as the temperature associated with the peak of $\tan$ $\delta$ ). It shows that the $T_{\mathrm{g}}$ of the nanofiber-reinforced epoxy matrix composites is higher than that of the neat epoxy. When attractive interactions are present at a polymer-nanofiller interface, confinement lead to enhancements rather than depressions in $T_{\mathrm{g}}$ relative to neat values $(33,34)$. At the interface, formed bonds restrain cooperative segmental mobility and lead to an increased $T_{\mathrm{g}}(35,36)$.

It is known that large surface area of the fillers and associated interfacial bonding play a significant role in enhancing mechanical properties of the multiphase, composite materials (37). The effectiveness of the nanofiber reinforcement is anticipated to correlate strongly with the quality of the interfacial bonding between the nanofibers and epoxy matrix. In support of this correlation, the sensitivity to the interfacial bonding was well-captured in the storage modulus data from DMA tests, by which the three choices of nanofiber surface chemistry/treatment were investigated. Table 1 also summarizes the storage moduli $\left(E^{\prime}\right)$ by DMA for the composites and the neat epoxy at 30 and $80{ }^{\circ} \mathrm{C}$. The complete temperature scans are also reported here in Figure 6. Upon closer examination, the results indicate that incorporation of $2 \%$ weight fraction of PSt nanofibers in epoxy was remarkably effective in increasing the storage modulus of the composite at $30^{\circ} \mathrm{C}$. There is more than a factor of 3 in improvement by PSt nanofiber reinforcement, in contrast with the neat epoxy. However, the influence of these fibers gradually decayed as the temperature was increased beyond the $T_{\mathrm{g}}$ of the composite. At $80{ }^{\circ} \mathrm{C}$, which is well above the curing and glass-transition temperature, the mean storage moduli of PSt/epoxy composite and the neat epoxy are about the same. The PSt nanofibers and epoxy both are of similar 
Table 1. Glass Transition Temperatures and $E^{\prime}$ Storage Modulus of Composites Incorporating Electrospun Fibers of $\mathrm{P}(\mathrm{St}-\mathrm{co}-\mathrm{GMA})$ with/without Ethylenediamine Spraying and PSt Compared to Neat Epoxy at 30 and $80^{\circ} \mathrm{C}$

\begin{tabular}{|c|c|c|c|c|}
\hline \multirow[b]{2}{*}{ Specimen } & \multirow[b]{2}{*}{$T_{\mathrm{g}}\left({ }^{\circ} \mathrm{C}\right)($ peak $\tan \delta)$} & \multirow[b]{2}{*}{ damping ratio $(\tan \delta)$} & \multicolumn{2}{|c|}{$E^{\prime}$ storage modulus (MPa) } \\
\hline & & & $30^{\circ} \mathrm{C}$ & $80^{\circ} \mathrm{C}$ \\
\hline neat epoxy & 60.1 & 0.679 & $1187 \pm 50$ & $130 \pm 3$ \\
\hline epoxy reinforced by $\mathrm{P}(\mathrm{St}-\mathrm{co}-\mathrm{GMA})$ nanofibers & 64.8 & 0.397 & $3825 \pm 100$ & $415 \pm 15$ \\
\hline epoxy reinforced by P(St-co-GMA)/EDA nanofibers (with cross-linker & 65.6 & 0.372 & $10038 \pm 100$ & $1570 \pm 15$ \\
\hline
\end{tabular}

agent spraying')

aromatic structures that can promote the interaction of the two materials. This results in the reinforcing effect provided by the PSt fibers. On the other hand, a significant downgrade in reinforcement at elevated temperature is attributed to the fact that PSt nanofibers and epoxy do not form strong chemical bonding or cross-linking across the interface, and its absence becomes further evident beyond the $T_{\mathrm{g}}$.

In contrast, the chemistry of P(St-co-GMA) fibers introduces an epoxide group that could react with the $\mathrm{NH}$ group in the hardener for epoxy resin, so that the stable supplementary cross-linking with the epoxy matrix is promoted. Outlook for the P(St-co-GMA)/epoxy composite is also similar at $30{ }^{\circ} \mathrm{C}$, but these surface-designed fibers appeared to preserve their contribution and influence in the storage modulus at elevated temperature as well. At $80^{\circ} \mathrm{C}$ beyond the $T_{\mathrm{g}}$ of the composite material, the storage modulus reached a plateau, where the increase was still around a factor of 3 compared to the neat epoxy. Comparison of the storage modulus curves associated with PSt and P(St-COGMA) fibers revealed that the benefit in the mechanical response due to presence of the fibers is preserved at elevated temperatures by supplementary GMA-epoxy interaction. To retain the high modulus even above the $T_{\mathrm{g}}$, enhanced adhesion between nanofiber and matrix is needed, as also observed in modified clay-epoxy nanocomposites (31).

The next question was whether the proven effect of nanofiber reinforcement with purpose-designed surface chemistry can be further enhanced, as far as the mechanical response is concerned. A stronger fiber-matrix interface was aimed by reinforcement of the PSt-co-GMA nanofibers, featuring epoxide rings in the surface chemistry and an additional process step of overcoating with the cross-linking agent ethylenediamine, before the resulting $\mathrm{P}(\mathrm{St}-\mathrm{co}-\mathrm{GMA})$ / EDA fibrous mats were embedded into the epoxy matrix. DMA results indicated that the storage modulus of epoxy reinforced with 2 wt \% mass fractions of P(St-co-GMA)/EDA nanofibers was about an order of magnitude higher than the neat epoxy (see Table 1 and Figure 6).

Cross-linking agent ethylenediamine applied by spraying over the fibrous mats introduced significant improvement on mechanical behavior because of epoxide ring-amine group interaction. It is attributed to increased cross-linking density by two mechanisms: (a) the nanofibers were themselves cross-linked, leading to an increase in inherent stiffness within the fibrous mat (15) (Figure 4) and, (b) the amine residue on the nanofiber surfaces reacted with the surround- ing epoxy matrix. As a result, the reinforcing effect of the $\mathrm{P}(\mathrm{St}-\mathrm{Co}-\mathrm{GMA}) / \mathrm{EDA}$ nanofibers was more than twice of the reinforcement by $\mathrm{P}(\mathrm{St}-\mathrm{CO}-\mathrm{GMA})$ nanofibers.

In addition, SEM micrographs in Figure 7A demonstrated that the fracture surface of neat epoxy was smooth and

A)

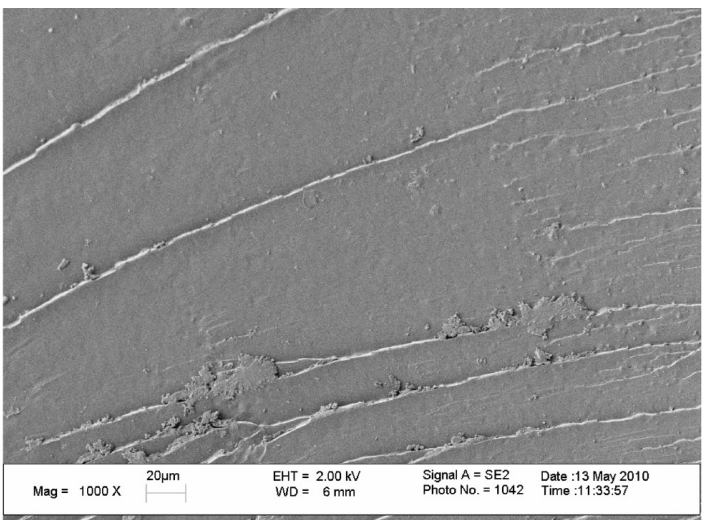

B)
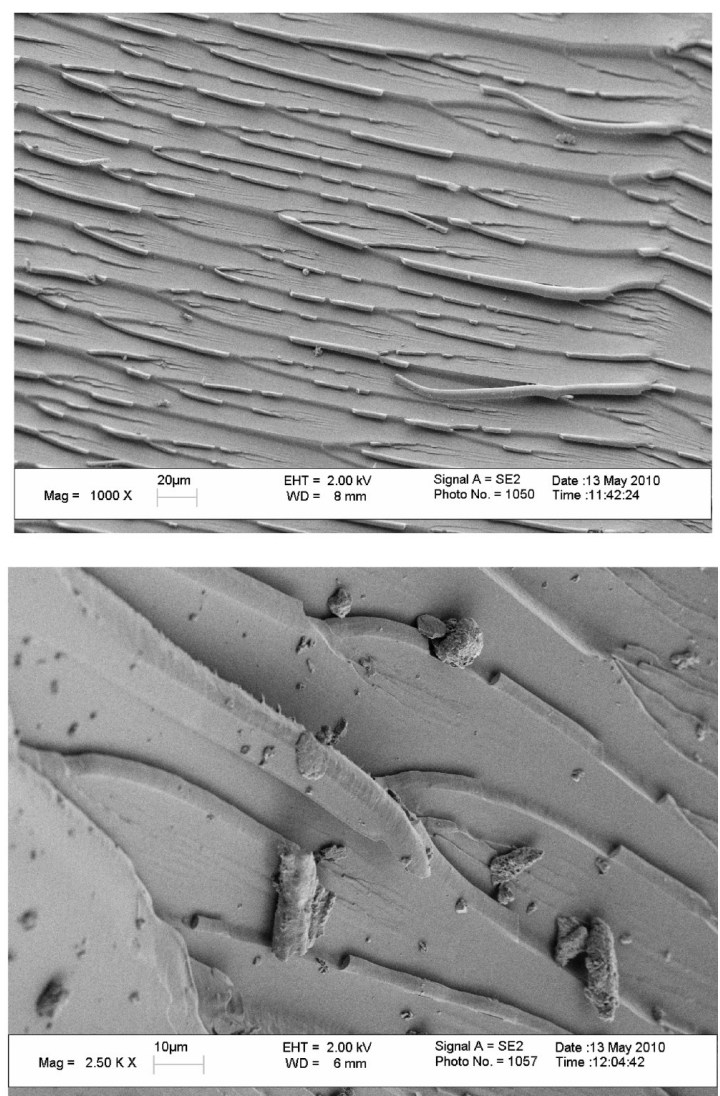

C)

FIGURE 7. SEM micrographs of fracture surfaces: (A) neat epoxy, (B, C) $\mathrm{P}$ (St-co-GMA)/EDA nanofiber-reinforced composites. 
consisted of large surface steps, as also observed by Fong (16) and Hsieh (38) et al. Nanofiber-reinforced composites, on the other hand, have numerous fracture lines in smaller steps that appear to be associated with the fiber distribution, as shown in Figure 7B. The rough fracture surfaces including fiber breakages are associated with the higher fracture energy in nanofiber-reinforced composites compared to the neat epoxy (38). Resistance to failure due to nanofibers can be explained by a "bridging mechanism" (39-41). When a microscale crack is initiated under flexural load, the surfacemodified nanofibers support the load and resist the crack opening, as shown in Figure 7C. As a result, the epoxy matrix is reinforced and toughened.

The flexural strength $\left(S_{\mathrm{F}}\right)$ and flexural modulus $\left(E_{\mathrm{Y}}\right)$ of the neat resin and nanocomposites containing a single layer of nanofibrous mat and the corresponding $0.2 \mathrm{wt} \%$ electrospun $\mathrm{P}(\mathrm{St}-\mathrm{co}-\mathrm{GMA})$ nanofiber-reinforced composites were also tested at room temperature. ASTM- D790 3-pointbending standard mechanical tests demonstrated that embedding a single layer of a PSt, P(St-Co-GMA), P(St-co-GMA)/ EDA nanofibrous mat increased the flexural modulus $\left(E_{Y}\right)$ by 23,27 , and $30 \%$ with respect to that of the neat epoxy. The flexural strength $\left(S_{\mathrm{F}}\right)$, when reinforced with $0.2 \%$ mass fraction of PSt, P(St-co-GMA), P(St-co-GMA)/EDA nanofiber, increased by 9,16 , and $23 \%$, correspondingly.

\section{CONCLUDING REMARKS}

Three different electrospun fiber chemistries were studied for their reinforcing abilities when embedded into epoxy resin. Specifically, PSt, P(St-co-GMA) and P(St-co-GMA)/EDA electrospun fibers were utilized. The near-room-temperature performances of PSt and P(St-Co-GMA) fibrous mats were quite similar, all showing a 3-fold increase in storage modulus compared to that of neat epoxy. Beyond the $T_{\mathrm{g}}$, the effect of PSt decayed and the reinforcing ability by P(St-co-GMA) was preserved. The performance of the cross-linked P(Stco-GMA)/EDA nanofibers, on the other hand, was far superior to composites of the other two fibers. Thermomechanical tests under flexural loads indicated that incorporation of lowweight-fraction ( $2 \mathrm{wt} \%$ ) $\mathrm{P}(\mathrm{St}$-co-GMA)/EDA nanofibers in epoxy is 10 and 2.5 times higher than neat and P(St-co-GMA) nanofiber-reinforced epoxy, respectively, even beyond the glass-transition temperature $T_{\mathrm{g}}$. The significant increase in the mechanical response is attributed to the combined effect of the two factors: the inherent cross-linked fiber structure and the surface chemistry of the electrospun fibers leading to cross-linked polymer matrix-nanofiber interfacial bonding.

Acknowledgment. E.Ö. acknowledges TUBITAK BIDEB graduate student scholarship.

Supporting Information Available: Differential scanning calorimetry results and wetting behavior of the electrospun PSt and P(St-co-GMA) fibers (drop shape analyses by water, epoxy resin, and cross-linking agent ethylene diamine)
(PDF). This material is available free of charge via the Internet at http://pubs.acs.org.

\section{REFERENCES AND NOTES}

(1) Beecroft, L.; Ober, C. K. Chem. Mater. 1997, 9, 1302-1317.

(2) Hussain, F.; Hojjati, M.; Okamoto, M.; Gorga, R. E. J. Composite Mater. 2006, 40, 17

(3) Gangopadhyay, R.; De, A. Chem. Mater. 2000, 12, 608.

(4) Okada, A.; Usuki, A. Macromol. Mater. Eng. 2006, 291, 1449 1476.

(5) Schmidt, G.; Malwitz, M. M. Curr. Opin. Colloid Interface Sci. 2003, 8, 103-108.

(6) Krishnamoorti, R.; Vaia, R. A. Polymer Nanocomposites; ACS Symposium Series; American Chemical Society: Washington, D.C., 2002; Vol. 804.

(7) Dzenis, Y. Science 2008, 319, 419-420.

(8) Lin, S.; Cai, Q.; Ji, J.; Sui, G.; Yu, Y.; Yang, X.; Ma, Q.; Wei, Y.; Deng, X. Comp. Sci. And Tech 2008, 68, 3322-3329.

(9) Uribe, A. R.; Arizmendi, L.; Guzman, M. E. R.; Guzman, S. S.; Silva, R. ACS Appl. Mater. Interfaces 2009, 11, 2502-2508.

(10) Kim, J. S.; Reneker, D. H. Polym. Comp. 1999, 20, 124-131

(11) Rasheed, A.; Dadmun, M. D.; Britt, P. F. J. Polym. Sci., Part B: Polym. Phys. 2006, 44, 3053-3061.

(12) Chen, G.; Liu, H.J. Appl. Polym. Sci. 2008, 110, 641-646.

(13) Gao, Y.; Sagi, S.; Zhang, L.; Liao, Y.; Cowles, D. M.; Sun, Y.; Fong, H. J. Appl. Polym. Sci. 2008, 110, 2063-2070.

(14) Dodiuk-Kenig, H.; Lizenboim, K.; Roth, S.; Zalsman, B.; McHale, W. A.; Jaffe, M.; Griswold, K. J. Nanomater. 2008, 1-6.

(15) Fang, J.; Lin, T.; Tian, W.; Sharma, A.; Wang, X. J. Appl. Polym. Sci. 2007, 105, 2321-2326.

(16) Fong, H. Polymer 2004, 45, 2427-2432

(17) Han, S. O.; Son, W. K.; Youk, J. H.; Park, W. H. J. Appl. Polym. Sci. 2008, 107, 1954-1959.

(18) Bergshoef, M. M.; Vancso, G. J. Adv. Mater. 2000, 11, 16.

(19) Chronakis, I. S. J. Mater. Proces. Technol. 2005, 283-293.

(20) Dagdeviren, C.; Papila, M. Polym. Compos. 2009, 1-9.

(21) Greiner, A.; Wendorff, J. H. Angew. Chem., Int. Ed. 2007, 46, $5670-5703$.

(22) Khojin, A. S.; Stone, J. J.; Zhong, W. H. J. Compos. Mater. 2007, 41.

(23) Zhu, J.; Peng, H.; Macias, F. R. Adv. Funct. Mater. 2004, 14.

(24) Suhr, J.; Koratkar, N. A.; Ye, D.; Lu, T. M. J. Intell. Mater. Syst. Struct. 2006, 17

(25) Jiang, L. Y.J. Adhes. 2010, 86 (3), 273-289

(26) Demir, M. M.; Menceloglu, Y. Z.; Erman, B. Polymer 2005, 46, $4127-4134$

(27) Yordem, O. S.; Papila, M.; Menceloglu, Y. Z. Mater. Des. 2008, 29, $34-44$

(28) Chandra, R.; Singh, S. P.; Gupta, K. Compos. Struct. 1999, 46, 41 51

(29) Zhou, X.; Shin, E.; Wang, K. W.; Bakis, C. E. Compos. Sci. Technol. 2004, 64, 2425-2437

(30) Rajoria, H.; Jalili, N. Comput. Sci. Technol. 2005, 65, 2079-2093.

(31) Ratna, D.; Chakraborty, B. C.; Dutta, H.; Banthia, A. K. Polym. Eng. Sci. 2006, 1667-1673.

(32) Chen, L.; Gong, X. L.; Li, W. H. Polym. Test. 2008, 27-3, 340 345 .

(33) Rittigstein, P.; Torkelson, J. M.J. Polym. Sci., Part B: Polym. Phys. 2006, 44, 2935-2943

(34) Ellison, C. J.; Torkelson, J. M. Nat. Mater. 2003, 2, 695.

(35) Frank, C. W. Science 1996, 273, 912.

(36) Priestly, R. D.; Ellison, C. J.; Broadbelt, L. J.; Torkelson, M. J Science 2005, 309, 456.

(37) Gawandi, A. A. Compos., Part B 2009, 40, 698-704.

(38) Hsieh, T. H.; Kinloch, A. J.; Masania, K.; Sohn Lee, J.; Taylor, A. C.; Sprenger, S. J. Mater. Sci. 2010, 45, 1193-1210.

(39) Marshall, D. B.; Cox, B. N.; Evans, A. G. Acta Metall. 1985, 23, 2013.

(40) Lawn, B. R. Fracture of Brittle Solids; Cambridge University Press: London, 1993.

(41) Crosby, A. J.; Lee, J. Y. Polym. Rev. 2007, 47, 217.

AM100288R

(1) 